\title{
Tangence
}

\section{Le livre du manuscrit inachevé ou Une saison dans la vie d'Emmanuel de Marie-Claire Blais}

\section{Jacques Cardinal}

Numéro 54, mars 1997

Poétique du Livre

URI : https://id.erudit.org/iderudit/025938ar

DOI : https://doi.org/10.7202/025938ar

Aller au sommaire du numéro

Éditeur(s)

Tangence

ISSN

0226-9554 (imprimé)

1710-0305 (numérique)

Découvrir la revue

Citer cet article

Cardinal, J. (1997). Le livre du manuscrit inachevé ou Une saison dans la vie d'Emmanuel de Marie-Claire Blais. Tangence, (54), 79-100.

https://doi.org/10.7202/025938ar d'utilisation que vous pouvez consulter en ligne.

https://apropos.erudit.org/fr/usagers/politique-dutilisation/ 


\section{Le livre du manuscrit inachevé ou Une saison dans la vie d'Emmanuel de Marie-Claire Blais ${ }^{1}$ Jacques Cardinal}

Tu ne l'ignores pas, mon cher frère, disait Jean Le Maigre d'un ton solennel, ma grand-mère me pousse vers le tombeau. Mais je songe à emporter avec moi mes ceuvres posthumes et celles qui ne le sont pas. Aussi, quand tu auras attaché mes bas avec des ficelles pour ne pas quils tombent et traînent derrière moi comme des ailes meurtries, va atu secours de mes poèmes, de mes romans, de mon ceuvre complète qui gémir - la pauvre sous tous les matelas de la maison, à cet enclroit que tu connais, sous les planches, clans les latrines. Ainsi épargné de la vengeance de mon père... 2

Un manuscrit. Un livre. Entre les deux, le temps et l'espace d'une parole qui, si elle a eu lieu, se cherche encore un lecteur. Entre le manuscrit et le livre un creux se dessine, entre-deux où une parole attend de se dire une deuxième fois (et peut-être enfin pour un nombre infini de fois). Cette seconde fois qui vient avec le livre est, dans cet après-coup, le re-commencement de l'écriture et d'une parole qui se risque sur la place publique. Elle commence en re-commençant. Double temps et double espace qui traverse tout le travail de l'écriture et celui de la lecture. Double scène aussi dont l'avant n'existe que dans le ressaisissement de l'après, par le livre. Car avant, l'écriture, en son état manuscrit, existe et n'existe pas; si elle est l'assomption et l'avènement

1 Cette étude s’inscrit dans le cadre d'une recherche subventionnée par le CRSH.

2 Marie-Claire Blais, Une saison dans la vie d'Emmanuel, Montréal, Boréal. coll. *Compact , 1991 (1965), p. 52. Toutes les citations renvoient à cette éclition; je n'indiquerai que le folio entre parenthèses. 
80

d'une parole et d'un sujet (lui-même divisé), elle est aussi menacée par l'oubli, l'indifférence, et la destruction sous toutes ses formes. On peut dire ainsi qu'entre le manuscrit et le livre règne l'espace-temps de la lettre en souffrance. De la lettre morte gisant dans les tiroirs de l'écrivain. Cet entre-deux montre toute la fragilité de la lettre (de l'écriture) dès lors qu'il n'y a ni personne ni une instance pour la transmettre aux autres. Lettre à demi morte en vérité puisque tout à la fois elle existe et n'existe pas... encore. Ainsi, le manuscrit est-il toujours à sauver de l'oubli, du désastre, de l'impuissance, de ce chant silencieux (qui peut être séduisant) pesant sur la lettre et, parfois, de la complaisance qu'il peut y avoir à se vautrer dans cette demi-mort. D'autres fois, faute d'éditeur, l'écrivain assume seul, extrême solitude et courage sans gloire, la sauvegarde de la lettre. Lui-même, à demi mort. Vivant cependant de continuer à écrire. Vivant d'une autre certitude. D'un autre temps.

Le manuscrit, c'est donc aussi l'antichambre de la parole en attente de l'autre. Et, même si l'écriture travaille à rejoindre cet autre (ce destinataire sans lequel la parole n'est sans cloute pas), à le nommer et à le symboliser, son geste est aussi lié à un autre, celui de la publication (du livre) qui l'accomplit d'une certaine manière pour en libérer l'écrivain. Manière encore d'assumer l'écrit, de le donner aux autres pour mieux se remettre à l'ouvrage. De l'autre à l'autre, l'écrivain donne à lire ce passage et cette adresse de la lettre. Il n'y a pas moins d'autre(s) ou d'altérité dans le manuscrit que dans le livre; mais sans la publication, cette adresse faite à l'autre reste comme en veuvage. Un manuscrit peut circuler sous le manteau, avoir ainsi des lecteurs et s'instituer autrement, cle manière informelle ou peut-être illicite. Mais c'est en règle générale par l'entremise de ce processus institutionnel qu'est la déposition légale d'un titre, d'un éditeur et d'un signataire, qu'un livre est reconnu et chose publique (quand bien même ce serait pour dire les errances et les incertitudes du sujet). La publication d'un livre implique donc ce geste institutionnel (le dépôt légal, le copyright) et le passage par la loi. Mais d'un autre côté, l'écrivain atteint-il vraiment ou toujours son destinataire là où l'écriture pose avec véhémence la question du désir et de l'altérité? Disons plutôt que l'institution du livre donne droit de cité à l'écriture (à son sujet), peu importe son adresse, sa demande, le vide, le manque ou le plein au nom duquel elle parle ou prétend parler. Elle fait circuler ou transmet ainsi tout autant 
l'incertitude que son contraire, selon les cas. Histoire de l'écriture. Vissicitudes de la lettre. Écrire. Cela suppose donc aussi qu'il y ait cet autre (ou plusieurs) pour que le livre advienne en tant que tel. Comme s'il fallait aussi, de la part de l'écrivain, le donner à l'autre pour que le geste même de l'écriture puisse s'accomplir. Encore. Enfin. Or, c'est de cela aussi qu'il s'agit dans le célèbre récit de Marie-Claire Blais. Récit de la transmission de la lettre, du livre et du désir de traverser le temp̀ps malgré la mort. Récit d'un manuscrit ayant promis le livre et qui, par magie, se trouve aussi à l'accomplir.

À relire l'exergue ci-dessus, on se souvient que le récit de Marie-Claire Blais est notamment celui de l'agonie de Jean Le Maigre, l'écrivain, le poète de la famille. Malgré cette agonie (et finalement cette mort) qui traverse en effet presque tout le récit, celui-ci est aussi le récit d'une cuuvre cherchant à survivre à cette fatalité. Récit d'agonie, il est aussi le récit de la lettre en souffrance et de la promesse du livre à venir. Sauver l'ouuvre, la sortir de l'espace familial qui ne la tolère pas, c'est donc vouloir inventer l'espace et le temps d'une autre parole par-delà une certaine impasse qui pèse sur le sujet. Impasse ou ratage qui concerne, on va le voir, le désir et l'héritage familial, aussi bien dire le nom et sa transmission. Sur le plan de l'imaginaire, ce ratage se déploie (se nomme) à deux niveaux; celui, d'abord, que l'on dirait d'un monde chimérique, frontière où justement l'humain et l'animal s'entremêlent soulignant en cela le difficile repérage du sujet dans la loi. L'autre aspect de ce ratage est lisible dans le discours sur la lettre (la parole, l'écriture, le livre) qui traverse tout le récit, discours qui est l'appel sublimé pour la maîtrise de l'univers symbolique et de la reconnaissance du sujet.

C'est cette traversée du désir que l'on peut suivre à la trace et dont l'objet est, ultimement, l'avènement de la parole et du livre. Faire en sorte que le livre advienne est sans doute la manouvre secrète de ce récit qui est, paradoxalement, celui d'un certain échec de l'écriture.

\section{Misère du désir (ou la famille-meute aux abois)}

Si l'on interroge l'histoire de cette famille depuis l'ordre du désir qui la détermine, on se rend compte qu'elle est celle d'un certain ratage. Cela est vrai d'abord de Grand-Mère Antoinette qui règne sur cette famille, se tenant au-dessus du silence résigné de 
la mère et de la brutalité intempestive du père. Cette impasse s'incarne dans cette femme toute-puissante, intouchable, mère et pourtant symboliquement encore vierge:

Ces vêtements de laine le séparaient [Emmanuel] encore de ce sein glacé qu'elle écrasait de la main d'un geste d'inquiétude ou de défense, car lorsqu'on approchait son corps étouffé sous la robe sévère, on croyait approcher en elle quelque frâtcheur endormie, ce désir ancien et fier que nul navait assouvi - on voulait dormir en elle, comme dans un fleuve chaud, reposer sur son coeur. Mais elle écartait Emmanuel de ce geste de la main qui, jadis, avait refusé l'amour, puni le désir de l'homme (p. 9).

Si elle a eu des enfants cependant, ce n'est que par devoir et respect de la religion. C'est donc d'abord ce ratage qui pèse sur la famille: impuissance du grand-père et frigidité de la grandmère. Ratage qui se transmet comme un héritage, celui notamment d'une solitude qui semble absolue, invincible et lourde comme une fatalité: "Oh! mon enfant, personne ne t'écoute, tu pleures vainement, tu apprendras vite que tu es seul au monde!" (p. 10). Sa fille, la mère d'Emmanuel, hérite, sur le plan du désir, de la même impasse. Cette frigidité qui semble se transmettre d'une génération à l'autre fait en sorte que la mère est en effet de glace devant son enfant qui n'incarne pas l'accomplissement de son désir : "Sa mère le prend dans ses bras. [...] Sa mère, elle, ne dit rien, ne répond plus, calme, profonde, désertée, peut-être. Il est là, mais elle l'oublie. Il ne fait en elle aucun écho de joie ni de désir. Il glisse en elle, il repose sans espoir. (p. 14). Ce désinvestissement ou cette désappropriation du sujet à l'égard de son enfant va si loin que c'est la grand-mère qui le nomme. Geste hautement symbolique puisque la nomination est pour ainsi dire paradigmatique de l'assomption (de la reconnaissance) d'un sujet par un autre. En usurpant la place des parents, Antoinette brouille ainsi le jeu de la filiation, le désir et sa loi. D'ailleurs, le père et la mère ne sont eux-mêmes jamais nommés; dès lors, comment pourraient-ils donner ce que, dans une certaine mesure, ils n'ont pas? Le repérage du sujet depuis sa position cedipienne est donc ici fort problématique. Jean Le Maigre dira lui aussi, à sa manière, ce ratage du désir parental:

La nuit, il dormait dans la même chambre que ses parents, séparé de sa mère par l'ombre de son père qui enveloppait d'une terreur sacrée ses rêves du présent comme ceux de l'avenir. Il reverrait plusieurs fois, en vieillissant, cette silhouette bru- 
tale allant et venant dans la chambre. N'était-ce pas lui l'étranger, l'ennemi géant qui violait sa mère chaque nuit, tandis qu'elle se plaignait doucement à voix basse (p. 127).

Cette vision d'une mère violée par son propre époux révèle l'échec, pour l'enfant, à symboliser sa propre place quant à la jouissance et à se saisir comme enfant désiré. Ce n'est donc pas l'amour qui se transmet dans ce désir, mais bien plutôt la haine, comme le dit Antoinette: "Et puis, je déteste les nouveau-nés; des insectes dans la poussière!» (p. 8). Imaginaire ici de l'infiniment petit, du presque-rien, du dégoûtant et de la mort. L'enfant, identifié à ce qui est au plus bas (ou presque) dans l'échelle de la vie animale, est dévalorisé et ramené à une forme de vie qui donne à penser d'emblée, outre la multitude indifférenciée, un modèle de reproduction où n'intervient pas le désir, lequel définit la dimension proprement humaine de la vie (et non pas l'instinct animal). Enfants-insectes, parasites, évoquant le registre imaginaire d'une multiplication aveugle, monstrueusement rapide et nombreuse. Multiplication qui donne à penser enfin la prolifération accélérée, la maladie, la peste, la mort. Or, la poussière c'est encore le déchet, ce surplus parasitaire et cette matière morte sur laquelle grouille le vivant indifférencié d'avant l'avènement et la reconnaissance du sujet. "Ca fourmille sur de la matière morte" serait peut-être ici la formule que l'on pourrait inventer pour dire la difficile symbolisation du désir d'une génération à l'autre. Ce ratage familial est lisible aussi dans l'exil de Jean Le Maigre et de son frère à l'orphelinat. Autre manière encore de dire l'enfant abandonné à lui-même (ou presque) qui traverse tout le roman. De même, lorsqu'Héloïse échoue au bordel, elle retrouve encore là une économie du désir qui n'est pas celle de la filiation et de l'assomption, mais plutôt celle, disons, de l'exploitation et de l'inconsêquence.

Ce ratage dans la transmission du désir est lisible dans le roman depuis cet imaginaire anthropozoomorphe qui le traverse du début à la fin. En effet, l'ambiguité qui pèse sur cette transmission fait en sorte que le sujet a du mal à se repérer depuis sa position oedipienne, qui suppose que le sujet est tout à la fois nommé et assumé par le désir parental en même temps que, par l'interdit de l'inceste, il assume sa castration comme moment structurant lui permettant d'élaborer sa place de désirant. Interdit qui, en le faisant sujet, le fait sujet de la loi. C'est l'effet de cette coupure qui marque son inscription dans le symbolique et lui 
84

permet de discerner lui-même, l'autre et son désir. Sans le repérage de cette coupure qui le discerne et lui permet en même temps de se lier à l'autre, le sujet erre dans un monde où prédomine un imaginaire chimérique dans lequel, justement, tout à la fois s'agglutine, se disperse, se mélange et s'efface $^{3}$. Imaginaire toujours plus ou moins effrayant des magmas et des chimères. Monde d'avant le monde, immonde, celui de l'indistinct où même le mort peut apparaître vivant et le vivant, mort. Problème de frontière, de limite et de discernement qui envahit tout dès lors que la coupure (la castration) ne fixe pas le sujet depuis son nom et ne règle pas le jeu de l'identité et de la différence, du même et de l'autre. Vertige de l'indifférencié puisque la loi, le tranchant de la loi, n'opère pas. Cette coupure depuis laquelle le sujet se repère est donc une sorte de seuil de l'humanité. C'est pourquoi, lorsque cette coupure est mal posée, le sujet erre dans un imaginaire animalisé sur ce bord soudain indiscernable. L'effet structurant de cette coupure est d'abord un fait de parole (de nomination), et c'est pourquoi elle peut tenir même si ce ne sont pas les parents biologiques qui la prononcent (bien qu'il reste toujours aussi dans ce cas à symboliser cette première perte). L'imaginaire du roman se donne ainsi à lire comme le symptôme d'une très précaire symbolisation du sujet à l'égard de la loi. Devenir-animal du sujet qui montre ce manque à symboliser le désir, la jouissance.

L'incipit du roman illustre bien d'ailleurs cette situation: "Les pieds de Grand-Mère Antoinette dominaient la chambre. Ils étaient là, tranquilles et sournois comme deux bêtes couchées, frémissant à peine dans leurs bottines noires, toujours prêts à se lever... "(p. 7). Ce corps animalisé on le retrouve sous toutes sortes de formes; ainsi, c'est Jean Le Maigre qui a "une agilité de renard" ( $p$. 21) ou qui marche "à quatre pattes sous la table. (p. 26) cherchant quelque nourriture * sa main tendue vers elle, comme la patte d'un chien" (p. 28). C'est aussi son corps continuellement parasité par les poux et les puces: "Les puces nous mangent, dit Jean Le Maigre, la vie est impossible. (p. 45). Plus loin, il se décrira comme une colombe (p. 39). Ou encore, écrivant un poème, c'est l'imaginaire du monde aquatique qui sou-

3 Quant à l'incidence de cette coupure ordonnatrice de l'avènement du sujet, je renvoie au livre de Denis Vasse, Lombilic et la dxix. Deux enfants en analyse, Paris, seuil, coll. - Le champ freudien*, 1974. 
tient cette fois l'image qu'il peut avoir de lui-même: "Ma tête est un aquarium où nagent les choses/Tes crimes et les miens/ Comme des chevaux de mers..." (p. 41). Or, ce corps s'animalisant, c'est aussi ce corps envahi par un autre, la maladie: "Tuberculos Tuberculorum, quel destin misérable pour un garçon doué comme toi, oh! le maigre Jean, toi que les rats ont grignoté par les pieds..." (p. 63). Le sujet est ici envahi de toute part par toutes sortes de bêtes qui tentent de le dévorer. Cet imaginaire de la dévoration s'inscrit symboliquement dans la problématique d'un sujet qui, mal nommé ou désiré, éprouve non seulement son corps (ou les limites de son identité) comme poreux, ouvert à toutes les altérations, mais qui perçoit aussi fantasmatiquement ses parents comme des ogres dès lors que l'assomption de l'enfant est ambiguë.

Grand-Mère Antoinette aussi s'animalise puisqu'elle est décrite soit comme "un corbeau" (p. 26) ou comme un chat sauvage: *... nous foudroyant de son regard d'orgueil, si bien que je ne savais plus qui était la bête féroce - le manteau de chat sauvage, ou ma grand-mère dans toute sa splendeur" (p. 76). Elle règne ainsi sur la famille comme le grand prédateur de la forêt. Ce manteau de bête est en effet le corps qui recouvre ou avale toute la famille-meute: "[...] elle pouvait loger toute une caravane d'animaux sous ce manteau de malheur! Enfin, une parole sensée, dit ma grand-mère qui abritait déjà Anita, et l'une d'autres des petites A, sous ses montagnes de fourrure. (p. 76). Cela montre aussi toute l'ambiguiité du personnage puisque, toute-puissante, on la craint, alors qu'elle est aussi protectrice (et qu'au lieu de la fuir on s'y colle). Cette ambiguité montre justement sa propre impasse quant au désir: elle est celle qui donne (qui protège), mais (souvent) à contrecouur parce que donner ce que l'on n'a pas soimême reçu est toujours difficile et suppose une abnégation qu'elle n'assume qu'à l'occasion. Cette ambiguité est donc celle d'un double langage, tour à tour maternant et dévorant, qui découle, on l'a dit, d'un certain ratage clans la transmission du désir. La parole familiale se règle ainsi sur cette double-contrainte (double bind) où le parent est tout autant tendre que brutal. Noeud d'amour et de haine. De folie aussi. Et presque inextricable. Là où GrandMère Antoinette est tout autant une mère nourricière et protectrice qu'une ogresse capable de dévorer les enfants.

Emmanuel, le nouveau-né s'animalise aussî̀ puisqu'il est décrit comme un *insecte. ( $p$. 8) et comme un "coquillage" 
86

(p. 9). Tout se passe, on le constate, comme si la frontière entre l'humanité et l'animalité était précaire: "Quel refuge, dans la chambre de Grand-Mère Antoinette, pour les jeunes garçons qui dormaient pêle-mêle avec le chat et le chien (et; quelquefois, un mouton...). (p. 128). Le lit est le lieu de tous les refuges et de toutes les confusions. De même, l'estomac de Pomme émet un tel bruit que l'on croit qu'il y a un ours dans la maison (p. 44). Donc, pas de distinction nette entre l'étable et la maison familiale, la ménagerie et la marmaille, la forêt (sauvage) et l'espace domestique. Pas de délimitation nette non plus, dans la chambre de la grand-mère, entre l'adulte et l'enfant. Or, ce règne du "pêlemêle", c'est justement le contraire d'un monde où le sujet symbolise sa différence et celle de l'autre depuis l'énonciation de l'interdit qui règle le jeu parental de la filiation. Ce "pêle-mêle est le signe de cette impasse qui pèse sur le sujet quant à la transmission du désir. Le Septième, enfin, n'est pas en reste dans cette métamorphose généralisée puisqu'il est aussi bien décrit comme une "chenille" (p. 67) ou un monstre (p. 22 et 66). Il grimpe dans les arbres comme un singe (p.92) et lorsque, sous l'influence de Jean Le Maigre, il se risque à écrire un poème, c'est encore l'imaginaire de la vie animale qui s'impose à lui : Dans la soupe que je mange/ je les vois, ils nagent, les poissons Les chats et les renards Au souvenir de ces meurtres/ je perds l'appétit..." (p. 41). Nous retrouvons là encore la grande scène de la dévoration, structurante d'un sujet mal nommé.

Si la chambre d'Héloïse est "infestée de rats " (p. 33) et que *le vieil harmonium [est] rongé par les rats...* (p. 67), c'est aussi la maison en elle-même qui, en tant que limite symbolique de l'espace familial, s'altère en étant dévorée par le parasite. Tout suggère ici un espace dont l'altération est négative puisqu'elle est la marque d'une impasse symbolique. Cette altération revient ainsi sur le mode imaginaire de la contamination ou de la maladie: "Car au printemps, chacun de nous bourgeonnait, fleurissait sous la vermine et la rougeole" (p. 67). Or, cette prédominance de l'imaginaire animal dans le récit se révèle également par l'effet de la mise en scène narrative. Nous entrons en effet dans cette maison par le bas ou par en-dessous, lieu ici du refoulement et de l'animalité. * Mais remuait encore sous la pointe de sa bottine une chose informe qu'elle tentait de repousser. Mon Dieu, une souris, un écureuil, il y a quelqu'un sous ma robe..." (p. 16) L'espace familial semble en effet coupé en deux: celui du haut 
où nous n'apercevons que des ombres de personnages adultes (espace qui serait ici celui d'un désir assumé), et celui du bas où grouille la meute d'enfants affamés, les rats et les puces. Et c'est d'ailleurs depuis cette vision au ras du sol que nous suivons le récit. C'est presque par le trou à rats que nous y entrons et qui, d'une certaine manière, animalise aussi le lecteur.

L'orphelinat est également un lieu où prédomine l'imaginaire de la vie animale. Dans leur cellule, Jean Le Maigre et le Septième sont comme dans "un piège à rats" ( $p$. 86) où grouillent des *araignées et mille choses grimpantes" (p. 86). La brutalité des comparses fait également en sorte que la vie y est pour ainsi dire en-dessous de l'humaine condition: "Ce n'était pas un orphelinat, c'était une jungle. Dans nos guenilles, les cheveux gluants de graisse sur les yeux, nous nous battions comme des animaux féroces dès que le directeur nous laissait seuls" (p. 89). C'est ici la loi de la jungle, celle du plus fort, qui nie l'espace social fondé sur le respect mutuel ou l'égale ctignité des sujets. Le directeur de l'orphelinat trône sur cette meute d'enfants avec son "regard fauve", (p. 85) incarnant l'ogre (p. 88), le dévoreur d'enfants. Enfin, l'usine où travaillent le Septième et Pomme (et de nombreux enfants) est décrite comme un lieu où l'on mène les a animaux à l'abattoir (p. 116). Machine à dévorer les enfants, encore. D'une institution à l'autre (de la famille à l'orphelinat à l'usine), on passe ainsi d'une scène à l'autre où l'enfant est en définitive soumis à la (menace de la) dévoration. À la mort.

Outre ce devenir-animal du sujet, c'est la famille en ellemême qui s'animalise en devenant meute:

Les voilà tous, les petits-enfants, les enfants, les cousins, les nièces et les neveux [...] sous les tables, sous les lits, ils me guettent de leurs yeux brillants dans l'ombre. [...] Ils ricanent, ils jouent avec les lacets de mes souliers. Ils me poursuivent toujours de ce ricanement stupide, de ce regard suppliant et hypocrite, je les chasse comme des mouches, mais ils reviennent, ils collent à moi comme une nuée de vermines, ils me dévorent... Mais Grand-Mère Antoinette domptait admirablement toute cette marée d'enfants qui grondaient à ces pieds (p. 11).

Car si la famille est cette institution à partir de laquelle un sujet advient par l'effet de la nomination (et ce que suppose ce geste quant à sa position à l'égard de la loi et du désir), on constate que c'est moins le règne de la singularité qui prédomine ici que celui de la multiplicité et de l'indifférenciation. La famille se 
88

transforme en meute, perdant le repère de son humanité. La famille-meute, c'est aussi le quasi effacement du nom propre dès lors que l'énumération et/ou la série remplace la singularité du nom. Ainsi, les filles de la famille sont-elles (presque toutes) non seulement animalisées, mais nommées justement comme les unités d'une série, celle de la lettre A: "Les Roberta-Anna-Anita avancèrent comme un lent troupeau de vaches... "(p. 42). Quasi effacement car un nom est là, mais il est pour ainsi dire pris déjà dans un pluriel, un ensemble ou un amalgame qui a pour effet de l'indifférencier parmi les autres. Cette façon de nommer montre ici la fragilité du processus nominatif et ce qu'il implique symboliquement. Ne sommes-nous pas ici dans les parages de l'archaïque puisque le seuil entre l'homme et l'animal est incertain? Incertitude qui est due, on l'a vu, au ratage du sujet à transmettre un désir et un nom porté par la jouissance. Il n'est pas insignifiant, à cet égard, de constater que les parents de Jean Le Maigre ne sont jamais nommés. Ce manque de nom ou ce nom manquant est aussi, pour le sujet pris dans cette filiation, le vertige sur lequel se fonde son manque-à-être et sa quête (sublimée) de la lettre.

Dès lors, ce qui règle ici l'espace domestique entre les sujets, c'est moins la parole (et son cortège de reconnaissances à l'égard de l'autre) que la faim, pulsion quasi première, comme si la loi était d'abord celle de la survie (animale): [...] tout ce déluge d'enfants, d'animaux, qui, plus tard, à nouveau, sortiraient de leur mystérieuse retraite et viendraient encore gratter à la porte pour mendier à leur grand-mère..." (p. 12) La faim semble d'abord dominer toute parole ou tout rapport à la parole, qui suppose une autre élaboration symbolique fondée sur le respect de l'autre ou le partage. Mais la faim est ici le commun dénominateur qui transforme les sujets en bêtes et les rameute autour de ce seul désir et de celle qui semble les dominer tous. Pas étonnant, dès lors, que le livre que lit Jean Le Maigre soit "rempli de repas fabuleux " (p. 25) car le livre est, non seulement lieu de l'évasion et de la souveraineté, mais promesse du repas fabuleux contre le règne de la faim et de la meute affamée. Le livre arrache ainsi le sujet à l'empire de la faim, de cette faim qui l'aveugle et le déshumanise.

Cette animalisation de l'espace familial, on peut donc la lire comme le symptôme d'un mal à symboliser sa place dans l'ordre de la filiation et du désir. Or, le désir de l'écriture (du livre) qui 
traverse le roman, on va le voir maintenant, vient aussi en réponse à ce ratage, à ce manque ou à cette trouée dans le désir parental.

\section{Misère de la lettre (ou le poème inachevé, dispersé, brûlé)}

Ce récit de Marie-Claire Blais n'est pas seulement celui d'une certaine misère familiale et sociale, il est aussi celui d'une misère de la lettre (de la parole, de l'écriture et du nom). Cette misère de la lettre est liée à celle du désir puisque le sujet, mal nommé, entend sublimer sa condition par l'écriture (la poésie, la prose), paradigme de la parole nommante. Misère de la lettre non seulement en raison du fait qu'elle est plus ou moins illicite (se cachant derrière la scène familiale et l'intolérance du père) mais aussi parce qu'elle n'est au fond que continuellement promise, s'avérant par là en perpétuel devenir, multipliant ses commencements mais demeurant toujours inachevée.

Disons d'abord que le livre (à lire, à écrire) est pour Jean Le Maigre un refuge; c'est à l'intérieur de lui qu'il se terre pour échapper à la famille, à la parole du père qui, lui, ne le tolère pas:

Ravi comme un prince dans ses vêtements en lambeaux, il se hâte de lire. Mon dieu que c'est amusant, dit-il en riant aux éclats. Tu as tort de rire, dit le père, je peux te l'arracher des mains, ce livre. [...] Il est trop tard, j'ai lu toutes les pages. On ne peut pas brûler les pages que j’ai lues. Elles sont écrites là ? (p. 18).

Le livre, la lecture, dessine cet espace souverain à l'intérieur de l'espace familial. C'est là que Jean Le Maigre se soustrait à la misère en se transformant, par la magie de la lecture, en prince souverain. La lecture est pour lui l'expérience de son inviolable intériorité. La lettre, gravée dans son âme, est indestructible. Jean Le Maigre ne résiste ainsi à son père qu'à se faire le corps-dulivre. Clandestin dans sa propre maison. Espace du repli où le sujet sublime la parole pour lutter contre les conditions d'existence quasi animales. Le livre creuse aussi un autre espace sous le terrier familial. Cet espace est celui de la parole, emblème de la souveraineté ou de l'assomption du sujet à l'égard du monde et des autres.

Si le livre est illicite dans la maison familiale, l'espace qui le tolère est toujours une cache, un en-dessous, un en-bas ou encore le lieu de l'expulsion ou du déchet: 
Enfermés dans les latrines, ils lisaient toute la bibliothèque du curé, écrivaient des poèmes d'une inspiration élancée, tel ce poème de Jean Le Maigre qui commençait et finissait ainsi: Combien funèbre la neige/ sous le vol des oiseaux noirs... (p. 30).

Le poétique (le religieux) et le scatologique, en principe opposés, s'entrecroisent ici pour laisser entendre le sort infamant réservé à la lettre et à ses équivalents symboliques. Autre lieu de mutilation ou de destruction de la lettre. Ainsi, lorsque par hasard Grand-Mère Antoinette trouve des poèmes, c'est pour aussitôt les brûler. Autodafé, encore: "J'ai écrit des poèmes. Il n'ajouta pas que sa grand-mère les mit au feu, le soir même, s'écriant que cela était scandaleux, que Jean Le Maigre irait en enfer, si choquée par le titre: À la chaude mâ̂tresse... qu'elle n'avait pas eu le courage d'aller plus loin" (p. 32). On le voit, le poème chante le désir, la jouissance; ce qui est d'une certaine façon intolérable sur la scène familiale. Or, chanter le désir, c'est justement la réponse de Jean Le Maigre, enfant malade, à ce silence mortifère quant à la jouissance qui pèse sur toute la famille.

Tout le récit est en quelque manière celui de la promesse de l'écriture. Ainsi, Jean Le Maigre ne cesse de (re)commencer son poème (ou son récit): "Tiens, je devrais écrire des poèmes." Il voyait déjà le titre: "Poème obscur écrit sur le dos de mon frère pendant son sommeil irréprochable (p. 46). Promesse des commencements, de l'œuuvre, du livre à venir. Ce poème en perpétuel devenir, il le rêve aussi :

Ah! Oui, je tombe dans un trou, l'eau est glacée. Je suis triste. Un aigle traverse le ciel. Je me noie ! Mais soudain, un vers superbe sort de ma bouche: Ô Ciel, d'un sombre adieu Je... Oup ! Je n’ai pas le temps de finir. Je disparais. Les ealux se referment ! Mains étrangleuses à mon propre cou, Oup! C'est fini. Je ne suis plus sur cette terre (p. 44).

Ce commencement et cette promesse s'abîment aussitôt, on le constate, dans la mort. L'émergence du vers et le silence de mort coïncident presque pour laisser ce début de poème orphelin. C'est là, d'une certaine manière, la scène emblématique de tout le récit. Une phrase (un vers) émerge, aussitôt absorbée par la mort. La mort règne sur ce continuel début d'écriture. Et la phrase (le vers) interrompue apparaît comme l'ultime trace de cette disparition. Par ailleurs, l'énoncé ("un vers superbe sort de ma bouche") peut s'entendre de deux manières selon justement 
le sens que l'on donne au signifiant "vers"; ainsi, si c'est bel et bien de poésie qu'il s'agit ici, il n'empêche que l'on peut entendre (et voir) l'animal ou l'insecte dégoûtant sortant de la bouche de Jean Le Maigre. Un même signifiant donc pour dire l'animalité, le bas, le dégoûtant, le déchet, la vomissure, l'abjection et, d'autre part, la forme sublimée de la parole (et du nom), soit la poésie, l'écriture. Cette association qui vient avec la lecture n'est pas arbitraire, non seulement parce qu'en l'occurrence le poète tombe ici dans un trou d'eau glacée, mais parce que l'isotopie animale est à ce point insistante qu'on ne peut que buter sur elle en le lisant. Cette ambiguité qui se donne à lire soudain par le jeu de ce signifiant montre justement le noeud qui noue les deux réseaux imaginaires qui traversent tout le récit. Le "vers", c'est tour à tour l'objet sublimé de la nomination et son contraire, l'objet chu de l'humanité, soit l'animal errant aux frontières du dégoût.

Ce commencement de l'écriture ne cesse de se relancer, de re-commencer tout au long du récit. Ainsi, un commencement de récit cherche à s'écrire sous nos yeux alors que Jean Le Maigre et le Septième espionnent leur sœur, Héloïse, la (supposée) dévote:

La visite chez Héloïse se transforma en roman qu'ils écrivirent à l'aube, entre le lit et l'armoire [...] enflammé par le beau titre inscrit dans son cahier: Journal d'un bomme à la proie des démons [...]. La piété d'Hélö̈se est donc le sujet de ce triste roman dont vous lirez la suite, chaque nuit, à la même heure, si les oiseaux de l'insomnie vous tourmentent comme ils me tourmentent! (p. 49)

On remarque là le désir de raconter l'autre, de le sauver et de se sauver en racontant son histoire édifiante. Écriture salvatrice qui, en nommant l'autre, l'arracherait, par sa magie symbolisante, à la misère de son désir. C'est aussi le fantasme d'un livre qui s'écrirait sous nos yeux, nuit après nuit, tel celui de Shéhérazade, pour conjurer la mort. D'un livre où se confondraient parfaitement - dans un pur présent - la vie et l'écriture. Ce fantasme, on doit peut-être le comprendre comme la volonté du sujet de cö̈ncider avec son nom, son désir. Rêverie autour d'une transmission de la lettre et ritualisation de l'écriture de la part du sujet comme pour s'enchaîner au temps, au sens, à la vie. Écrire donc, malgré la mort, le sommeil (ou l'interruption, sous toutes ses formes):

Le lecteur peut me suivre dans mon douloureux pèlerinage vers la mort, la forêt s'épaissit, mes yeux se ferment: Et je vieillis de 
mille ans $\dot{A}$ ma solitude songeant. -Je dois donc suspendre ici, la palpitante histoire d'Héloïse. Pour plus de détails, attendez jusqu'a la semaine prochaine. Ayant levé les yeux de mon cahier, je viens dapercevoir mon frère, hélas foudroyé par le sommeil et qui gît, la face contre terre... Je vais moi-même défaillir sur le sol dans quelques instants et me servir du coude de mon frère comme oreiller. Que le lecteur veuille bien pardonner mon absence. Ma gorge brûle, mes reins chancellent, mon genou fléchit, et de mon nez douloureux... Jean Le Maigre s’était endormi (p. 49-50).

Le ton ironico-cynique semble dire, "la littérature; faute de mieux", ou le leurre qui ne leurre qu'à moitié, mais dont on ne peut faire l'économie. Cette autre promesse d'écriture, ce n'est encore toutefois que du temps gagné sur la mort. Cette promesse de l'écriture, on la retrouve encore alors que le poète séjourne au noviciat: *Jean Le Maigre avait l'intention d'écrire lui-même une vie de saint devenu pécheur, pour édifier ses camarades * (p. 60). Mais déjà la mort est proche, et cette promesse de l'écriture (du poème, du récit) se dissémine dans tous ses (re)commencements, faisant de son cuuvre une ceuvre fragmentaire, inachevée et dispersée, cachée dans tous les coins de la maison (dont elle voudrait ébranler les fondements). Déjà la mort est proche et l'œuvre perpétuellement (re)commencée n'a plus d'autre temps que pour celui du testament: "Pieds nus dans le courant d'air, Jean Le Maigre buvait du laid chaud en songeant qu'il était temps pour lui d'écrire son testament au Septième et de choisir le lieu où sa grand-mère l'enterrerait." (p. 62). Et c'est ce que nous lègue ce récit, le journal d'un mourant, d'un poète mort trop jeune pour écrire autre chose que son agonie et la promesse de l'œuṿre: "Maintenant cloué à son lit par l'ordre du docteur et la complice sollicitude du Frère Théodule, Jean Le Maigre écrivait tristement son autobiographie... " (p. 63). Récit qui a déjà valeur de testament. Voix d'outre-tombe, presque. Mais que peut donc léguer ce sujet qui n'écrit que la promesse du livre à venir? Quelques vers, un journal..., c'est bien peu. Mais ne lègue-t-il pas surtout le désir du livre ou sa promesse, ce qui est tout? Livre de la promesse. Livre de l'attente. Livre du deuil.

Ce livre qui est promesse du livre c'est aussi, dans le récit, la lettre qui n'atteint jamais son destinataire. Ainsi en est-il des lettres que Jean Le Maigre écrit à Mademoiselle Lorgnette, son institutrice: "Fier comme un coq je laissais traîner partout dans la maison mes versions grecques, mes éloges funèbres, mes fables et mes tragédies quand je découvris que mon père les faisait disparaître à 
mesure dans les latrines. Quelle déception! "(p. 77). Il en va ainsi aussi des lettres destinées à Grand-Mère Antoinette: "J'écrivais de nombreuses lettres à ma grand-mère que $M$. le Directeur déchirait à mesure en souriant. (p. 90). L'écriture, la promesse de l'écriture, est continuellement interrompue ou détruite:

Dans les plis amers de ma retraite, j'écrivais de fiévreux poèmes que ma grand-mère brûlait à mesure lorsque son tremblant regard tombait sur les mots passion et amour et luxure. Elle coupait toujours le cou au mot luxure, mais le mot honneur lui arrachait des soupirs de satisfaction (p. 92).

Machine à dévorer la lettre, encore. Autodafé. Et c'est là aussi le malheureux destin d'Héloïse dont les lettres n'atteignent pas davantage leur destinataire:

[...] et sur le plancher sale, un crucifix, des lettres, des monceaux de lettres qu'Héloïse n'avait jamais eu le courage d'envoyer à ceux à qui elle les avait écrites, et qu'elle se lisait à elle-même silencieusement aujourd'hui, dans la solitude de sà chambre (p. 110).

Lettres promises. Lettres de l'amour, de la promesse de l'amour. Lettres en souffrance. Lettres mortes.

Et ce n'est pas seulement l'écriture de Jean Le Maigre qui ne cesse d'être détruite sous nos yeux, c'est aussi tout le registre de la lettre (y compris son corps) et de ses équivalents symboliques qui sont menacés ou altérés par la destruction. Ainsi, par exemple, les fautes de français de l'institutrice de même que ce dictionnaire mutilé et par conséquent incomplet: "Je ne connaissais pas incangru ni arosoire et j'usai mes yeux à parcourir l'unique dictionnaire de l'école qui s'arrêtait à la page 122 à la lettre $\mathrm{H}$ " (p. 78). Ce dictionnaire, pour ainsi dire coupé en deux, c'est déjà le symbole d'un certain état de la lettre et du livre en tant qu'ils sont inachevés et dispersés. Il ne faut donc pas s'étonner si "[1'] école tombe en morceaux" (p.82) et sera incendiée (p. 85) par Jean Le Maigre et son frère. Destruction de la lettre, du livre, de la parole en même temps que se multiplient les esquisses, les brouillons, les commencements... Double geste - presque simultané - qui accompagne le sujet de cette mé-prise de la lettre et du désir. Passant d'un autodafé à l'autre. D'une promesse à l'autre. Livre de l'indestructible promesse.

Condamné à mort et conscient de l'être, Jean Le Maigre sait qu'il écrit le récit de son agonie; il sait aussi que ce qu'il écrit ne 
sera peut-être lu qu'après sa mort, imminente: "Ah! Si tu crois, dit Jean Le Maigre, si tu crois que je m'en irai au paradis tout doucement, comme ça, avec une bénédiction! [...] J'ai une idée, dit-il, je vais faire mon ceuvre posthume!» (p. 38). Qu'à l'âge de l'adolescence, le poète proclame déjà son "ceuvre posthumen, voilà qui souligne ici la fragilité de la lettre, de l'écriture, de l'ceuvre. Que toute ouvre soit d'emblée liée aussi à la mort de l'écrivain, et sous toutes sortes de formes et de tons, on le sait. Mais que le sujet d'une ouvre, à peine existante, (se) promettant de s'accomplir chaque fois qu'elle commence une phrase, s'anticipe déjà depuis la mort, d'outre-tombe, cela suppose une autre économie de l'écriture. "CEuvre posthume. faite de commencements et de recommencements épars et inachevée. Comme une écriture qui se serait arrêtée sur le seuil, ne serait que le seuil, qu'une première phrase brusquement interrompue par un silence de mort. On peut d'ailleurs voir dans ce récit une structure formelle entièrement déterminée par le jeu incessant de l'interruption, de l'inachevé, de l'avorté, du fragmentaire, de la dispersion et de la dissémination. C'est tout autant le poème que récite en hoquetant le Septième (p. 24) que tous ces débuts de poèmes et de récits qui ponctuent l'ensemble. Mais surtout, c'est la mort (annoncée) de Jean Le Maigre, qui rythme le jeu de cette interruption, de cet inachèvement. Livre qui bat au rythme du glas.

Si l'on considère le récit formellement, et dans son ensemble, on remarque d'abord qu'il s'articule sur deux trames emboitées l'une dans l'autre: celle de la voix narrative, et celle du poème, du récit, de l'écriture en somme (dont Jean Le Maigre est la figure éponyme) et qui apparaît par l'usage fait de l'italique. L'italique produit une sorte de trouée ou un pli dans le récit. Cette trouée est aussi bien une irruption qu'une interruption dans le fil du récit et peut se lire également comme un effet de dispersion ou de dissémination de la lettre, promise, toujours à venir. Outre les nombreuses italiques qui scandent la narration, le pli se gonfle dans le récit tout particulièrement alors que nous lisons le journal ou l'autobiographie de Jean Le Maigre. Situé en plein centre du roman (p. 6393), ce journal, "ceuvre posthume" fait pli dans le récit avant que la voix narrative ne prenne le relais et le sauve en quelque sorte de la mort ${ }^{4}$. Mais que reste-t-il de ces cahiers après la mort du poète?

4 Formellement, ce récit pose également un problème de genre. Elaine D. Cancalon, dans son article, - Une saison dans la vie d timmanuel: le discours 
Ces cahiers de la promesse de l'écriture, toujours déjà testamentaires, il revient notamment à Grand-Mère Antoinette d'en être, à la fin, la gardienne :

\begin{abstract}
Ouvrant un à un les minces cahiers d'écolier, elle vit aussi les lettres que Jean Le Maigre avait tracées avec application, application et désespoir, car certains mots avaient perdu de leurs syllabes lorsqu'une main soudain languissante s'était interrompue au milieu d'une phrase, d'un paragraphe. Chaque cahier trahissait un moment de la maladie de Jean Le Maigre, une arcleur heureuse et triste, sur le point de se tarir. Grand-Mère Antoinette eût voulu serrer contre son coeur ces pages, afin que chacune s'inscrive en elle pour toujours avec sa morsure frầche, son secret féroce (p. 106).
\end{abstract}

Elle est la récitante de l'ouuvre interrompue, inachevée. Le manuscrit est à la fois vivant et mort; il vit dans la voix de GrandMère Antoinette, mais il est mort puisqu'il n'est pas encore dans l'espace public du livre. Récitation qui sauvegarde apparemment l'œuvre; mais la transmet-elle? Pas vraiment; elle s'y vautre plutôt amoureusement comme si ce corps mort était sa chose. Le manuscrit, en définitive, elle l'avale et le dévore (quand bien même ce serait par amour). Elle devient en quelque sorte gardienne du tombeau de Jean Le Maigre: "Elle les traînait partout avec elle dans la crainte qu'une main ingrate les jette au feu" (p. 116). Cette main qui brûlait les poèmes de Jean Le Maigre les protège maintenant sans les transmettre. Renversement où se

du conte. (Voix et images, n' 43, automne 1989, p. 102-110) a montré, en s'appuyant sur Bakhtine, le plurilinguisme et l'intertextualité de ce roman. Elle note, avec pertinence, l'incidence du conte de fées (* Hansel et Gretel *, soit l'imaginaire de l'ogre et du monstre), et de la référence plutôt ironique au discours du poète maudit (Rimbaud étant ici l'incarnation du jeune poète). À cela, on pourrait ajouter Lautréamont. Manière de rappeler aussi que le livre est aussi le livre de ses citations. Gilles Marcotte, dans Le roman à l'imparfait (Montréal, l'Hexagone, coll. *Typo*, 1976/1989) considère aussi que dans ce roman nous sommes du côté du conte, c'est-à-dire du côté de l'imaginaire de l'enfant trouvé/perdu. Il note cependant avec perspicacité qu'il ne s'agit pas là simplement, pour le sujet, du règne souverain de son imaginaire (déniant le réel), mais que, dans ce cas: „L'enfant trouvé sait qu'il écrit. [...] ... écrivant, il arme le rêve, en fait l'instrument d'une intervention ravageuse dans l'ordre des choses* (p. 140). C'est dire à quel point, le texte de Marie-Claire Blais est aussi un hybride de conte et de roman. C'est dire aussi comment cette ouvre se développe en cherchant à symboliser (à assumer) la place de l'écrivain. Les manuscrits de Pauline Archange (1968) témoignent de ce cheminement, du manuscrit à l'avènement du livre. 
remarque encore l'ambivalence fondamentale à l'égard de la lettre.

Il n'y a pas que Grand-Mère Antoinette qui se vautre sur le corps mort du poète. C'est aussi le cas du Frère Théodule, l'infirmier du noviciat qui l'a accompagné vers la mort. Lui, aussi, se vautre de manière quasi nécrophilique sur le corps d'écriture du poète décédé:

Sa fine main blanche, croyant toucher le chaud cadavre de Jean Le Maigre, effleurait du bout des doigts le rugueux papier aux lettres enfantines dont les $\mathrm{G}$, les $\mathrm{L}$ et les $\mathrm{C}$, et toute lettre ronde et fraîche évoquait pour le pauvre Frère des formes connues, des sensations intimes. [...] Les $\mathrm{A}$, les $\mathrm{V}$, les $\mathrm{L}$ et les $\mathrm{U}$ avaient le frémissement des joues que caresse une brise tiède et automnale... (p.120)

Ce qui est lu, c'est moins un récit, un poème (qui rappelle vaguement le Sonnet des voyelles de Rimbaud), qu'un morcellement symbolique du sujet, son éparpillement, sa dispersion corps et lettres. Fétichisme de la lettre qui efface en même temps l'énoncé, le discours, l'adresse du texte vers l'autre. Le corps de la lettre, dans sa graphie, est là tout son sens replié sur luimême, sur son fantasme. Lettres-corps; blason du poète mort. Sans l'enchaînement des mots et des phrases, ne reste plus que la lettre détachée de son dire. Démembrement du nom et de la phrase. CEuvre de mort, aussi. Sublimation de la lettre et destruction du nom. Geste ambivalent où se remarque le désir de sauvegarder le corps du poète (ses lettres), mais en détruisant son nom, sa phrase, son vers. Cette ambivalence montre le double jeu d'un sujet aux prises avec un difficile travail de symbolisation de la lettre. Le frère Théodule, surnommé Théo Crapula, est d'une certaine manière un thanatologue doublé d'un écrivain, rédigeant les épitaphes de ses enfants-adolescents morts trop tôt (p. 120). Ogre-thanatographe, il rédige en quelque sorte le livre des morts. Mais si l'épitaphe sauvegarde le nom, c'est le récit dans son ensemble qui en transmet la mémoire. Le livre est donc ici encore, et malgré les autodafés, l'instance qui sauvegarde le nom en le racontant. Il fait de ce qui ne pourrait être qu'un livre d'épitaphes (suite de noms monumentalisés), un livre de la mémoire du nom. Et c'est la voix narrative du roman, voilée dans sa convention tutélaire, qui accomplit ce passage. Pour en faire aussi un livre de deuil. Stèle sur laquelle le nom traverse la mort. Un livre. 
Inventer le passage de la lettre à la phrase au récit (au nom), c'est d'ailleurs justement ce que fantasme Jean Le Maigre dans une scène hautement symbolique que l'on dirait être celle d'un père qui ne serait pas destructeur de la lettre (de la non-transmission du nom), mais dépositaire enfin d'une parole:

Mais le Septième et moi avions beaucoup de respect pour l'beure de fumerie de mon père. [...] Nous regardions jaillir de la fumée ces lettres géantes, les o, les 1 et les c (et quelques notes de musique) que mon père formait de sa bouche ignorante, lui qui ne pouvait pas lire son nom, même écrit en majuscules. Notre père écrivait ainsi des romans, des contes qu'il ne lirait jamais, car de sa pipe sortait l'illustration brumeuse de nos cuvres futures. C'est ainsi que je devins un poète (p. 71).

Père analphabète, incapable de lire son nom, mais qui apparaît là transfiguré comme le plus lettré des sujets. Fantasme donc d'un père porteur d'une lettre (un récit) et du désir qu'elle incarne. Non pas qu'il ne transmette rien; au contraire, il transmet son silence et sa brutalité. Mais le père qui s'imagine ici au détour du récit, c'est celui de la parole, garante de sa place dans l'économie du désir. Mais la lettre ici ne s'envole-t-elle pas déjà en fumée? Elle brûle encore dans le fourneau de la haine que porte le père à la lettre. Sans doute; mais c'est là aussi lire le bûcher du livre comme son contraire, autel sublimé de la lettre. Donc: lire le père malgré lui, l'inventer, le réinscrire dans l'ordre de la lettre et du nom qui est celui, symbolique, de sa place 5 . Jean Le Maigre invente ainsi le père parlant (nommant) pour se donner une filiation et un devenir dans les lettres. Double geste où s'inscrit la reconnaissance du sujet. Le roman s'avère en cela la mise en scène de ce difficile nouage entre le nom (la filiation) et une vocation d'écrivain, d'homme de lettres.

Malgré cette misère de la lettre (et du désir), le récit n'est pas platement misérabiliste. Il n'est pas non plus et simplement l'envers d'un certain roman de la terre, célébrant les valeurs traditionnelles du Québec. Non. Ce qui apparaît nouveau dans ce récit, c'est ce mélange étrange d'assujettissement et de souveraineté. Car ce qui donne ce ton au livre, c'est ce Jean Le Maigre parfaitement souverain dans sa place de poète; libre, sans culpa-

5 Voir à ce sujet, Guy Rosolato, "Du père *, Essais sur le symbolique, Paris, Gallimard, coll. *Tel ", 1968. 
bilité et sans ressentiment, il incarne le poète qui est aussi au-dessus des misères de son milieu. Car si la mort peut interrompre son ceuvre, la misère n'altère en rien son désir de se reconnaître poète. Jean Le Maigre est vivant d'une certitude que la mort n'ébranle pas et que la folie n'atteint pas. Il ne se morfond pas dans l'impuissance. Il chante plutôt le désir infini de la parole et du nom (non celui de sa démission). Nous sommes en un sens loin ici du récit du poète mélancolique qui doute de sa voix, de son désir et de sa valeur (si fréquent, par ailleurs, dans la littérature québécoise $)^{6}$. Au contraire, l'inébranlable souveraineté de Jean Le Maigre affirme le désir de parole malgré toutes les vicissitudes de la vie familiale et sociale (et politique). C'est pour ainsi dire cette souveraineté, cette force affirmative qui traverse le récit et lui donne ce ton unique entre la continuelle destruction de la lettre et la certitude triomphante de la vocation du poète. Si Jean Le Maigre meurt trop jeune (il est tué par la pauvreté), cela n'altère en rien son essence ou son identité qui est une vérité de naissance ("Dès ma naissance, j'ai eu le front couronné de poux! Un poète, s'écria mon père, dans un élan de joie" (p. 63)). En un sens, et malgré le continuel autodafé qui le traverse, le récit affirme que le poète est ce phénix qui perpétuellement peut renaître de ses cendres. Si la mort traverse tout le récit, ce dernier n'est pas platement morbide, mais célèbre dans le même souffle la voix immortelle du poète (de l'écrivain) qui chante le monde. Malgré le glas qui scande le récit, celui-ci n'est-il pas en effet le récit de la naissance (dont Emmanuel est ici l'emblème) ou de la vie (toujours) recommencée? Noeud de vie et de mort.

Orphée, donc. Encore. Livre où le sujet passe par l'enfer, le royaume des morts et revient à la vie. Livre où s'affirme encore la souveraineté du poète sur le monde. Livre infini et continuellement recommencé s'écrivant sur la cendre des autodafés.

6 On pense ici à une certaine filiation du poète maudit québécois de Crémazie à Gauvreau en passant par Nelligan et Saint-Denys Garneau. Pierre Nepveu a analysé (dans son livre, L'écologie du réel, Montréal, Boréal, coll. "Papiers collés*, 1988) cette filiation et son envers, de même que l'opposition prose/poésie sur laquelle elle repose. 


\section{Tombeau de l'écrivain (ou le récit du manuscrit testamentaire)}

$\mathrm{Si}$ ce récit édifie le tombeau du poète mort trop jeune, il garde vivante, ce faisant et malgré cela, la place du poète. Sauvegarde qui est l'ultime triomphe d'un monde qui ne le devient que par cette voix. Souveraineté de la vocation du poète (de l'écrivain) malgré le feu. La vocation demeure, phénix qui traverse le bûcher pour affirmer le désir de la lettre et sa transmission. Jean Le Maigre meurt, tout comme Léopold son frère aîné avant lui, mais la chaîne de la vocation poétique ne se rompt pas (ne brûle pas); elle traverse souverainement l'autodafé. Cette souveraineté traverse le roman; la voix narrative voilée dans le récit est cette voix souveraine qui nous transmet, même mutilé ou en cendres, le récit toujours à venir (renaissant) de la lettre. Souveraine, parce qu'elle ne meurt pas et que ne meurt pas le désir de réciter (de raconter, de symboliser), quand bien même ce serait le récit de la lettre mutilée. La souveraineté, c'est le désir sans cesse renaissant qui saisit le sujet de se dire (de s'écrire), de se raconter en surmontant le malheur. Tombeau de l'écrivain, oui. Mais "tombeau chantant" si l'on peut dire où la parole n'est pas ce silence de mort pesant sur le sujet, mais parole transmise, édifiée, reconnue dans le cycle de la vie et l'ordre de la nomination.

On a vu qu'Une saison dans la vie d'Emmanuel est aussi le récit du manuscrit inachevé et dispersé. Livre de la promesse du livre. Livre à venir. Il est aussi le récit d'une mort annoncée ou qui ne cesse de s'écrire pour se donner à lire comme telle. La voix narrative qui prend le manuscrit en charge est donc son tombeau (son témoignage). Récit s'édifiant sur le corps morcelé d'une ouvre en voie d'être posthume. Cette double trame, c'est celle aussi entre le livre et l'écriture manuscrite. À ce niveau, la voix narrative fait, de ces fragments de récits agoniques et testamentaires, un livre. Nous lisons, paradoxalement, le récit d'un manuscrit qui vraisemblablement le restera à jamais. Mais cela, nous l'oublions justement alors que nous lisons son histoire. Nous lisons le manuscrit et l'histoire de ce perpétuel manuscrit sans qu'aucun narrateur ne revendique de le sauvegarder ou de le publier. Voix narrative fantomatique, drappée dans la convention immémoriale de l'art de raconter qui agit comme le passeur du manuscrit, et nous enchante ou nous fait croire à la pérennité du geste de raconter. Cette voix se lève au moment même où le désastre de la lettre et du désir (du livre) semble presque total. 
100

C'est elle qui transmet et sauvegarde cette parole du poète agonisant sur son ouvre. La voix narrative prend le relais pour nous transmettre le récit, et le sauver à son tour.

Magie de l'écriture. Là où la littérature est aussi, par excellence, le lieu de la sauvegarde du sujet (de sa mémoire et de son nom) contre l'oubli, le temps, la mort. 